\title{
Abnormal Behavior Monitoring By Using RNN For 5G Networks In Smart Cities.
}

\section{Theresal Thangappan}

SRM Institute of Science and Technology https://orcid.org/0000-0003-2164-2208

Supratik Sengupta ( $\nabla$ supratik.tukai@gmail.com )

SRM Institute of Science and Technology https://orcid.org/0000-0002-0232-2981

\section{Rahul Tank}

SRM Institute of Science and Technology

\section{Piyush Jain}

SRM Institute of Science and Technology

\section{Research Article}

Keywords: wireless communication, propagation, legitimate, data transfer

Posted Date: June 3rd, 2021

DOI: https://doi.org/10.21203/rs.3.rs-498283/v1

License: (9) This work is licensed under a Creative Commons Attribution 4.0 International License. Read Full License 


\title{
Abnormal Behavior Monitoring by using RNN for 5G Networks in Smart Cities
}

\author{
Theresal Thangappan ${ }^{1 *}$, Supratik Sengupta ${ }^{2}$, Rahul Tank $^{3}$, Piyush Jain ${ }^{4}$ \\ College of Engineering, SRM Institute of Science and Technology, Kattankulathur, India - \\ 603203 \\ 1*theresal2004@gmail.com, ${ }^{2}$ supratik.tukai@gmail.com, ${ }^{3}$ rahultank2001@gmail.com, \\ ${ }^{4}$ piyushsrm6065@gmail.com
}

\section{ABSTRACT}

The use of mm Wave or millimeter wave bands for systems based on mobile and wireless communication's next generation is a promising alternate that has the capability for supporting continuous rates of data over tens of Gigabits per second or Gbps. Although the advantages are clear of using a large available spectrum, there are some challenges which are related to the characteristics of propagation at higher frequency bands which are needed to be dealt. In the existing system, an adaptive transmission that can reduce the probability of outage of secrecy capacity primarily when the mean signal to noise ratio of the eavesdropper is comparatively lower than the legitimate receiver's mean signal to noise ratio. As more number of devices are connected to the network, security threats are also becoming a real concern. For ensuring the normal operation of smart city, monitoring of abnormal behaviors becomes essential. A smart city requires a guarantee mechanism which is reliable for guaranteeing the QoS. The network's QoS or quality of service can be affected by various abnormalities in the network like abnormal flow, link fault and DDoS attack etc. which causes packet loss and also interruption of service. Also as more number of IoT devices are connected to the network, security threats are the main issue as more personal information becomes vulnerable.

In the proposed system, Design of Resilient or Spatial Correlation is evaluated, this kind of Spatial Correlation enable the physical later to do the iterative search for $\mathrm{N}$ number of times until the communication is established without any blockage of objects. To reduce the disconnection of data transfer due to line of sight issues, an adaptive Resilient Correlation scheme is newly proposed here. 


\section{INTRODUCTION}

The fifth generation multipurpose organization is $5 \mathrm{G}$. After the organizations of $1 \mathrm{G}, 2 \mathrm{G}, 3 \mathrm{G}$, and $4 \mathrm{G}, 5 \mathrm{G}$ is the new global standard. $5 \mathrm{G}$ can also empower one more organization which is proposed to connect together everything that includes various items, gadgets and machines. [1]

$5 \mathrm{G}$ remote invention is envisioned that it will be conveying information speeds of multi-Gbps, will have extremely low latency, more dependability, and large capacity of network, extended availability, with a better experience to a greater number of clients. The better efficiency and superior performance enables new client meetings and connect new undertakings. [2]

In cell architecture, land territory is partitioned into various cells each with its radio service. In AMPS the territory is huge and in digital services the zone is small in comparison. Ordinarily cells are of hexagonal shape. Every cell utilizes a frequency range that isn't utilized by its adjoining cells. In any case, frequencies might be reused in non-contiguous cells. [3]

In the cell-less plan, a mobile terminal can decide to access at least one or more than one base station or access points by various downlinks and uplinks with consideration about the status and demands of the wireless channel, or decide not to access any base stations when the versatile terminal is inactive. Which means, a versatile terminal won't connect with any base station prior to communicate information. In this case, base stations do not have to keep a list of related mobile terminals; all things being equal, the SDN regulator chooses which at least one base station play out the transmission of information by the control link for the mobile terminal. [4]

As the number of breaches continue to increase, the security of $5 \mathrm{G}$ has become an issue that is more important than ever. To a network a huge threat is posed by the IoT devices. The use cases of 5G, like devices related to healthcare, autonomous driving as well as smart homes makes more personal data accessible to the attackers as ever. The network of $5 \mathrm{G}$ should be architecture to developing to increasing security needs. End-to-end security is required the $5 \mathrm{G}$ that uses its architecture that is software-defined to automatically identify as well as alleviate the threats. [5]

For getting the best performance out of the current technologies that are wireless like Wi-Fi, Bluetooth, Zigbee,3G, 4G etc., IoT services make trade-offs in performance. Unlike these, the design of the 5G networks will be bringing for massive Internet of Things the required performance level. A perceived fully universal connected world will be enabled by this.

An RNN or recurrent neural network is a part of artificial neural networks where associations in between the nodes structure, along a temporal sequence, a directed graph. This permits it to show temporal behavior 
which is dynamic. Gotten from feedforward neural networks, recurrent neural networks can utilize their memory or internal state so as to handle input sequences of variable lengths. This creates them pertinent for errands like unsegmented as well as connected recognition of handwriting or acknowledgment of speech.

A generalization of the feed-forward neural network which consists of an internal memory is the recurrent neural network. It performs the same function for each and every data input and hence is recurrent in nature whereas the current input's output is dependent on past one computation. The output after being produced is copied and directed back into the recurrent network. It considers the current input and the output that has been learned from the last input, for making a decision. [6]

For finding the shortest path for establishing a connection between two nodes, Dijkstra's Shortest Path Algorithm is used. Dijkstra's shortest path algorithm is used in this proposed project for establishing the connection between the nodes and their corresponding shortest distance node. If any blockage is found, a new route is selected and Hilbert Correlation is performed. Then the new route is selected as the final route between the given two nodes. Spatial autocorrelation's measure define to what degree values at spatial locations, are similar to one another. Hence, two things are needed by us: locations and observations.

A smart city is a city that utilizes innovation to offer services and take care of city issues. A smart city does things like improve transportation and availability, improve social administrations, advance manageability, and give its residents a voice. [7]

Smart cities utilize a blend of the Internet of things or IoT gadgets, solutions regarding software, and user interfaces (UI), and networks of communication. In any case, they depend above all else on the IoT. The IoT is an organization of connected gadgets - like vehicles, sensors, or appliances of home - that can communicate and exchange information. Information gathered and conveyed by the IoT sensors and gadgets is put away in the cloud or on servers. [8]

Smart cities are the development patterns of future urban areas, which include numerous parts of everyday life in urban communities, including e-business, intelligent systems of transportation, telemedicine, management of metropolis, security surveillance, management of logistics, community services, social networks, etc. To prepare for services mentioned above, smart cities are utilizing different networks and technologies of wireless communication, which includes ZigBee, Bluetooth, RFID wireless innovations, remote cell organizations, remote neighborhood (WLANs), and networks for radio broadcast, networks of wireless sensors and body area networks, with numerous more. The given technologies related to wireless communication alongside networks of fiber communication and networks of cable, structure the pervasive smart city's networks. 
Smart City's essential objective is to establish an environment that yields a great life to its occupants while additionally creating generally speaking economic development. Along these lines, a significant benefit of smart cities is their capacity to encourage an expanded delivery of services to its residents with less framework and cost. [9]

Albeit a carrier for transmissions of service for smart cities has already been found, the smart city additionally needs a guarantee mechanism which is reliable to guarantee the QoS. The QoS in network is influenced by some abnormalities in a network. Abnormality recognition, additionally called outlier detection, is the identification of unexpected events, observations, or things that vary essentially from the standard. [10]

\section{LITERATURE SURVEY}

Tao Han ET. Al. [4] proposed networks of 5G converged cell-less communication for smart cities bearing in mind the distribution of ultra-dense $5 \mathrm{G}$ wireless networks. SDN controllers are configured for the management of traffic scheduling and resource allocation. The results of simulation shows that the probability of coverage is improved in the converged cell-less communication network. Also the saving of energy at both the mobile terminals and BSs are enhanced.

Chao Li ET. Al. [10] presents a technology for monitoring of abnormal behaviors using Spearman Correlation Coefficient and based on recurrent neural network in 5G Network. A smart city requires a guarantee mechanism which is reliable for guaranteeing the QoS and thus ensuring a smart city's stable operation. The simulation shows parts of network where the true value differs from the predicted value and those are the parts where some abnormality is present.

Jose Santos ET. Al. [11] presents a solution for anomaly detection for the applications of Smart City is offered, that focuses on the low-power solutions of Fog Computing and estimated in the range of Antwerp's testbed of City of Things. The result obtained shows that Birch clustering as well as RC outlier detection of anomaly mechanisms, both can be done by fog resources near the IoT sensors and, through this, timely alerts will be sent when something unusual is detected.

Christophe Croux ET. Al. [12] presents the studies on the robustness of non-parametric estimators of correlation such as the Kendall as well as the Spearman correlation by means of their gross error sensitivities and influence functions. The estimator of the Pearson correlation, at normal distribution, has the highest efficiency, but on the other hand the statistical efficiency of Kendall as well as Spearman estimators of correlation is always more than 70 percent. 
Alex Sherstinsky [13] reviews the challenges which are faced when the standard RNN network is trained. The challenges are addressed by the transformation of Recurrent Neural Network into "Vanilla LSTM" network using a set of rational arguments. All the equations that are related to the LSTM system are provided along with the thorough explanations of the entities that are its constituent.

Ke Xiao ET. Al. [14] proposed a new method for prediction of the moving unmanned aerial vehicles trajectory so as to for the BS to perform a more exact beamforming and reduce the unnecessary capacity loss. The simulation results have shown that predictor that is RNN-based has high accuracy of prediction that is of average $98 \%$ and hence the BS can perform a more precise beamforming which reduces the unnecessary capacity loss.

Imad Alawe ET. Al. [15] proposes scaling techniques which are based on the neural networks for forecasting the loads which are upcoming. The new architecture or the NGC architecture can address the flexibility and the scalability by the introduction of more modular NF or Network Functions for composing the services of the control plane. The simulation results display the robustness of this scheme when compared with a technique for scaling which is threshold based.

Anastasios Zafeiropoulos ET. Al. [16] states that by means of part of 5G PPP Initiative, the 5G Architecture Working Group looks to realize the 5G architecture by capturing the main technological enablers and new trends. Within the 5G system, the major pillars for supporting the heterogeneous KPIs in a way that is cost efficient of the new use cases are architecture which is service-based, end-to-end (E2E) network slicing, SDN and NFV.

Lijun Wang ET. Al. [17] proposes a scheme of communication for vehicular 5G networks, where access points that are moving are installed on vehicles for enabling the user of the vehicle's access. The results from simulation shows that the system which is proposed reduces the latency by a considerable amount, with the improvement of the vehicular network's connectivity.

Tareq M. Shami ET. Al. [18] states that for achieving a balance among the radio resources' SINR gain and loss, finding an optimum power level difference (PLD) value is crucial which is able to identify under CoMP mode how may users can operate. Hence a scheme for radio resource management is provided which is able to support cell-less architecture. Results have demonstrated that the algorithm proposed, in comparison to static clustering performs better in terms of the energy efficiency. 


\section{ABNORMALITY DETECTION DUE TO LINE OF SIGHT BLOCKAGE}

In the proposed system, Design of Resilient or Spatial Correlation is evaluated and this kind of spatial Correlation enable the physical receiver to do the iterative search for $\mathrm{N}$ number of times until the communication is established without any blockage of objects. To reduce the disconnection of data transfer due to line of sight issues, an adaptive Resilient Correlation scheme is newly proposed here.

Dijkstra's shortest path search algorithm has been used here to find the shortest distance nodes. In case any blockage is present in the route between two nodes, a new route will be selected. Hilbert Correlation will be performed on the new route and then this new route will be made as the final route.

The Simulations are done in three modules:

\section{- Module 1: Design of wireless sensor networks}

This module contains the creation of nodes randomly in the free space, allocating the energy and distance cost for the nodes. Finding the cluster head on different environment will be the next task.

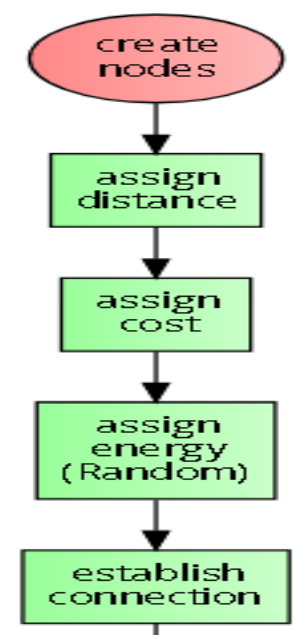

Fig 1. Module 1: Design of wireless sensor networks

\section{- Module 2: Communication Establishment}

Once the nodes are aligned and created, the next job is to establish the connection in the network, the established nodes connect with the shortest distance nodes after the cluster and tries to find out the Blockage in the path. 


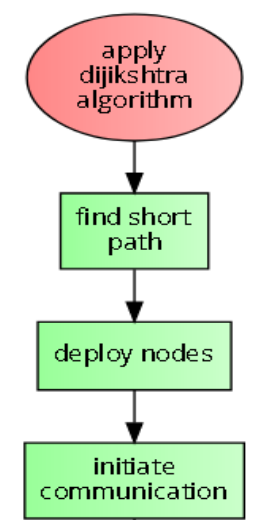

Fig 2. Module 2: Communication Establishment

\section{- Module 3: RNN for Abnormality Analysis}

Among a received signal's average gain and the signal's arrival angle, spatial correlation suggests that there is a correlation. Spatial correlation is decreased by rich multipath propagation, by means of spreading the signal so as the components of multipath are received from various different spatial directions. The integration module presents the blockage removal and establishment through spatial checking the maximum correlation point at the input data and reflected data through recurrent neural network.

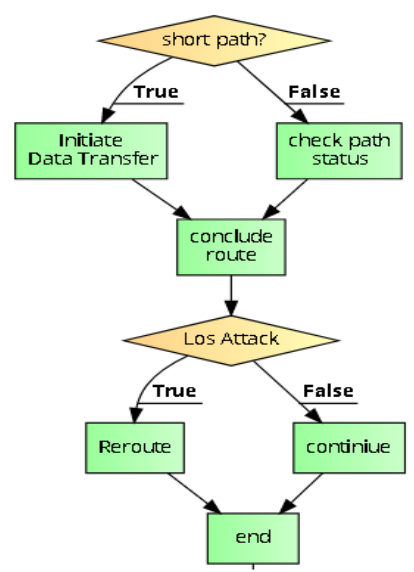

Fig 3. Module 3: RNN for Abnormality Analysis 


\section{RESULTS AND ANALYSIS}

The simulations are done in the MATLAB R2017b Software.
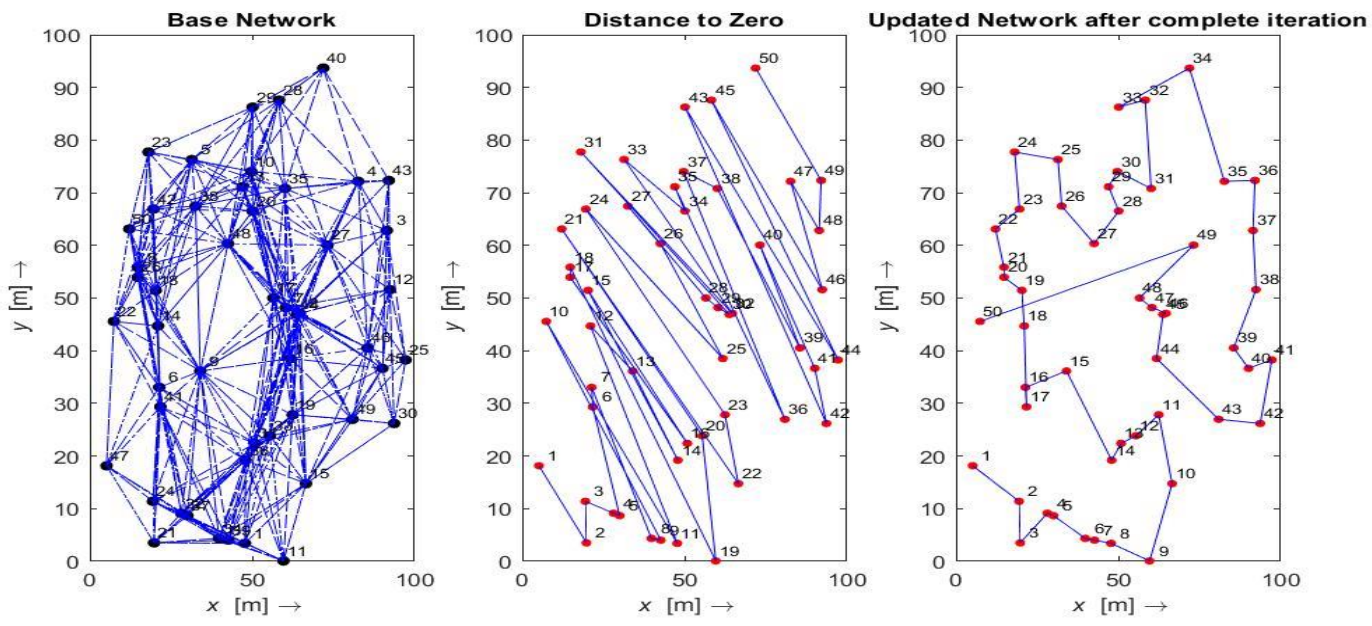

\section{Figure 1. Process of Node Connectivity}

The nodes are deployed in the free space. Figure 1 shows the process of node connectivity after the creation of the nodes and the assignment of the distance, cost and energy to the nodes. The base network is created when all the nodes in a given space are connected to some other nodes randomly. The figure also shows Distance to Zero where the node nearest to the origin is taken as 1 , the node next nearest to the origin is taken as 2 and so on. The established nodes then connect with the shortest distance node and the updated network after complete iteration is shown. Dijkstra's shortest path algorithm has been used to connect to shortest distance node.

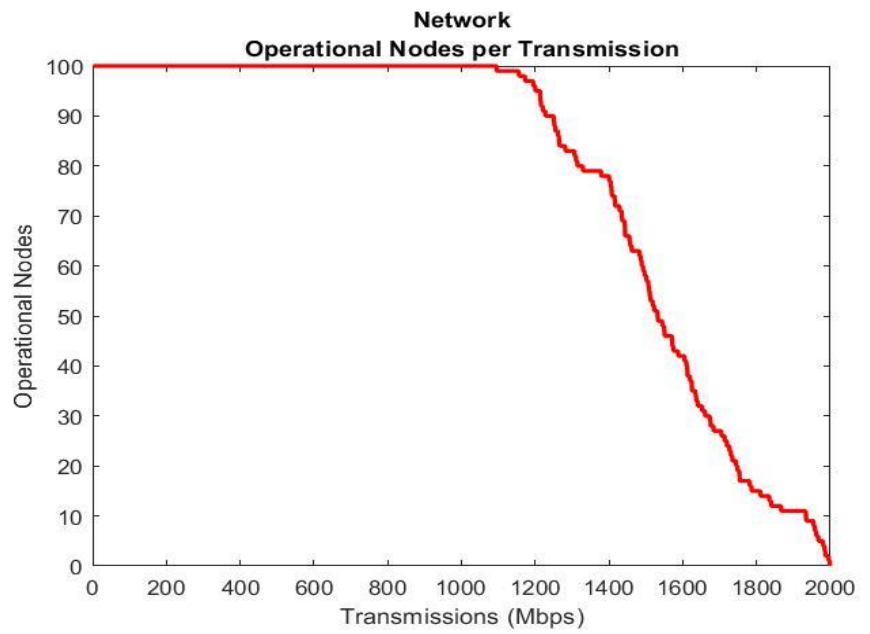

Figure 2. Network Operational Nodes per Transmission 
Figure 2 shows the Network Operational Nodes per Transmission. Some of the nodes may have the energy for the data transfer and some nodes may not have the required energy for data transfer. With more number of iterations the number of nodes that are operational reduces. Hence more and more nodes become nonoperational.

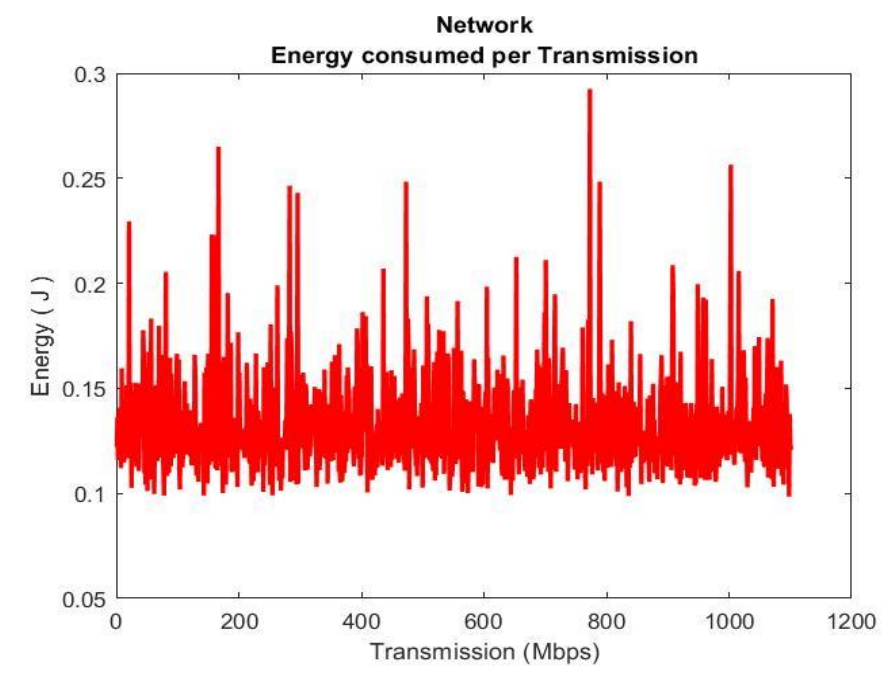

\section{Figure 3. Energy Consumed per Transmission}

Each node is assigned a random energy while creating the nodes and before the establishment of the connection. Figure 3 shows the Network Energy consumed per transmission. The figure shows that with each transmission, random energy is consumed.

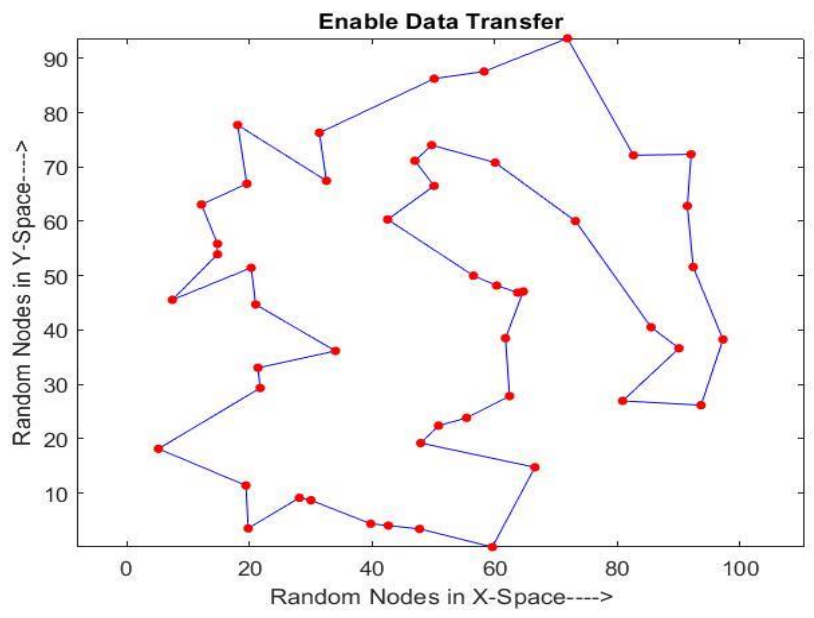

Figure 4. Enable Data Transfer 
After the assignment of distance and random energy to the nodes, when the nodes are ready, they transfer the data. Some nodes may have the energy required for the transfer of data and some nodes may not have the required energy. Figure 4 shows the enabling of data transfer which is based on the energy of the nodes.

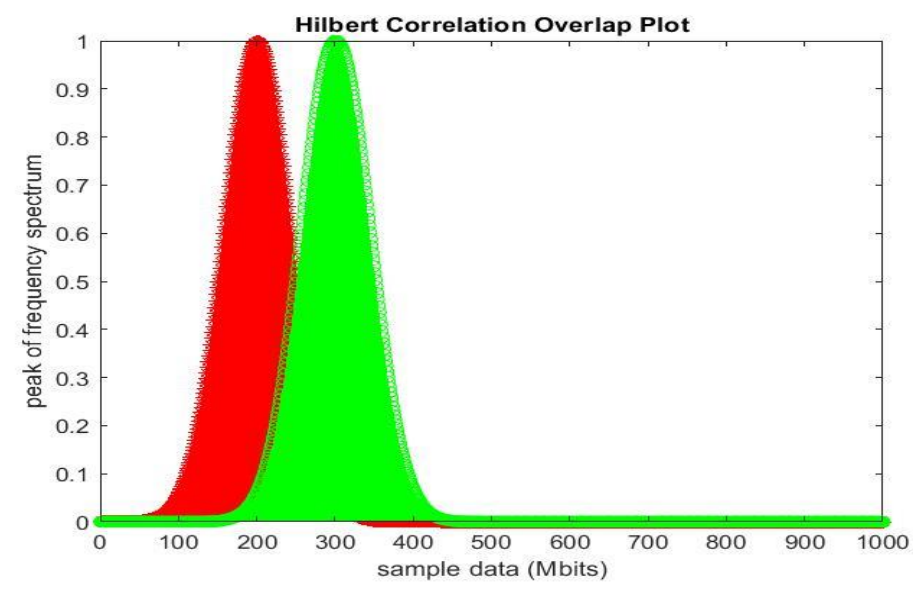

\section{Figure 5. Hilbert Correlation Overlap Plot}

Figure 5 shows the Hilbert Correlation Overlap Plot. The correlation is performed between the input signal and the reflected signal from a node. This correlation enables the receiver to do the iterative search for $\mathrm{N}$ number of times until the communication is established without any blockage of objects. Input signal is shown by green curve and reflected signal is shown by red curve.

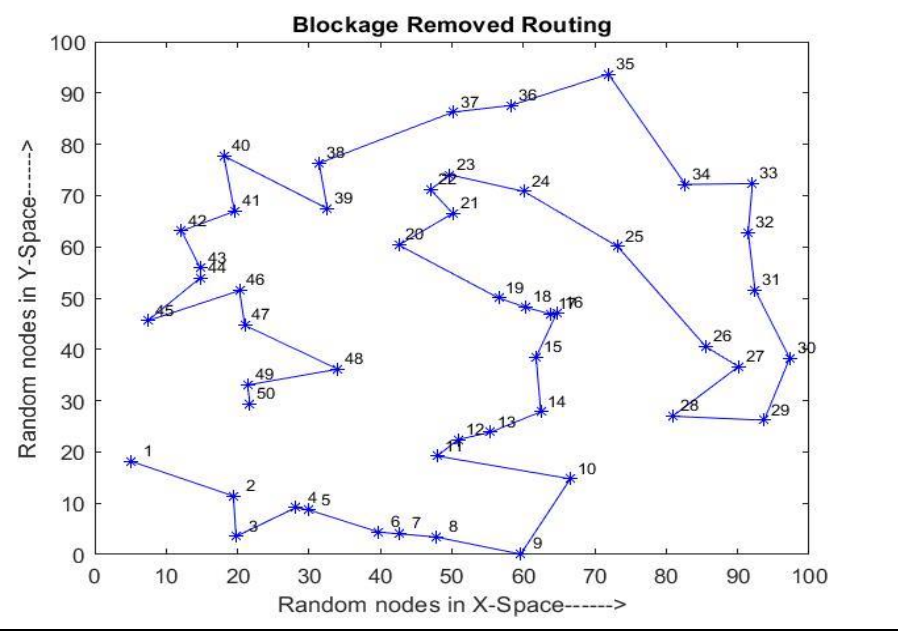

\section{Fig 6. Blockage Removed Routing}

Figure 6 shows the Blockage removed routing. Whenever there is a blockage found in the route between two nodes, a new route will be selected by the nodes that will be free from any LOS blockage. The Hilbert 
correlation will be calculated for the new route, and if it is found to be good, then the new route will be made final.

\section{CONCLUSION}

As demand of high data speed is increasing along with the number of subscribers, the technologies at present such as 3G, 4G are unable in supporting this and therefore there comes a necessity for the development for mobile network's next generation that is the $5 \mathrm{G}$ network. As more number of devices are connected to the network, security threats becomes a major issue as more personal data becomes vulnerable. For ensuring a smart city's normal operation, abnormality detection is necessary. The proposed system focused on spatial correlation based line of sight problem would be solved. The proposed system uses Dijkstra's algorithm for shortest path search. The proposed framework uses adjustable routing model through spatial correlation and abnormality detection through RNN network is developed. The proposed technique reduce disconnection of data transfer as line of sight problem was evaluated. The result shows the proposed rerouting consumes less delay and the propagation of data packets would be altered.

\section{DECLARATIONS}

Funding- Not Applicable

Conflict of Interests/ Competing Interests- Not Applicable

Availability of data and material- Not Applicable

Code Availability- Not Applicable

\section{REFERENCES}

[1] The Concept of Fifth Generation (5G) Mobile Technology by Khalid A. Fakeeh, 2015.

[2] High-reliability and Low-latency Wireless Communication for Internet of Things: Challenges, Fundamentals and Enabling Technologies by Zheng Ma, Ming Xiao, Yue Xiao, Zhibo Pang, H. Vincent Poor and Branka Vucetic, 2020.

[3] Advanced Mobile Phone Service: The Cellular Concept by V. H. Mac Donald, 1979.

[4] 5G Converged Cell-Less Communications in Smart Cities by Tao Han, Xiaohu Ge, Lijun Wang, Kyung Sup Kwak, Yujie Han, and Xiong Liu, 2017. 
[5] Privacy and Security Issues in the 5G-Enabled Internet of Things by Yulei Wu, Haojun Huang, Cheng-Xiang Wang and Yi Pan, 2019.

[6] A Critical Review of Recurrent Neural Networks for Sequence Learning by Zachary C. Lipton, 2015.

[7] Everything You wanted to Know about Smart Cities by Saraju P. Mohanty, Uma Choppali, Elias Kougianos, 2016.

[8] Smart Cities and the Internet of Things by Robert R. Harmon, Enrique G. Castro-Leon, Sandhiprakash Bhide, 2015.

[9] Smart Cities: Engaging users and developers to foster innovation ecosystems by Rodger Lea, David Vogt, Mike Blackstock, Nam Giang, 2015.

[10] Spearman Correlation Coefficient Abnormal Behavior Monitoring Technology Based on RNN in 5G Network for Smart City by Chao Li, Hui Yang, Bowen Bao, Huifeng Guo, Yong Jiang, Jie Zhang, 2020.

[11] Anomaly detection for Smart City applications over 5G Low Power Wide Area Networks by Jose Santos, Philip Leroux, Tim Wauters, Bruno Volckaert and Filip De Turck.

[12] Influence functions of the Spearman and Kendall correlation measures by Christophe Croux, Catherine Dehon, 2010.

[13] Fundamentals of Recurrent Neural Network (RNN) and Long Short-Term Memory (LSTM) Network by Alex Sherstinsky, 2020.

[14] Trajectory Prediction of UAV in Smart City using Recurrent Neural Networks by Ke Xiao, Jianyu Zhao, Yunhua He and Shui Yu, 2019.

[15] Smart scaling of the 5G core network: an RNN-based approach by Imad Alawe, Yassine Hadjadj-Aou, Adlen Ksentini, Philippe Bertin, Cesar Viho, and Davy Darche, 2018.

[16] 5G PPP Architecture Working Group: View on 5G Architecture by Anastasios Zafeiropoulos, Anastasius Gavras, Anna Tzanakaki, Antonino Albanese, 2019.

[17] Cell-less Communications in 5G Vehicular Networks Based on Vehicle-Installed Access Points by Lijun Wang, Tao Han, Qiang Li, Jia Yan, Xiong Liu, and Dexiang Deng, 2017.

[18] User-centric JT-CoMP clustering in a 5G cell-less architecture by Tareq M. Shami, David Grace, Alister Burr, Muhammad D. Zakaria, 2018.

[19] Artificial Intelligence-Defined 5G Radio Access Networks by Miao Yao, Munawwar Sohul, Vuk Marojevic, and Jeffrey H. Reed, 2019.

[20] Edge Computing in 5G: A Review by Najmul Hassan, Kok-Lim Alvin Yau, Celimuge Wu, 2016. 
[21] When 5G Meets Deep Learning: A Systematic Review by Guto Leoni Santos, Patricia Takako Endo, Djamel Sadok and Judith Kelner, 2020.

[22] Deep Learning at the Mobile Edge: Opportunities for 5G Networks by Miranda McClellan, Cristina Cervelló-Pastor and Sebastià Sallent, 2020.

[23] SDN for 5G by Maede Zolanvari, 2015.

[24] Smart Campus to Smart City: 5G SDN-based Networking for Enhanced Urban Living by Stefano Basagni, Kaushik R. Chowdhury, Miriam E. Leeser, and Tommaso Melodia.

[25] Millimeter Wave Mobile Communications for 5G Cellular: It Will Work! By Theodore S. Rappaport, Shu Sun, Rimma Mayzus, Hang Zhao, Yaniv Azar, Kevin Wang, George N. Wong, Jocelyn K. Schulz, Mathew Samimi, And Felix Gutierrez, 2013.

[26] Security Analysis of Mobile Device-to-Device Network Applications by Kecheng Liu ; Wenlong Shen ; Yu Cheng ; Lin X. Cai ; Qing Li ; Sheng Zhou, 2019.

[27] Challenges of Using MIMO Channel Technology in 5G wireless Communication Systems by Dr. Intisar Al-Mejibli, 2013.

[28] Large-scale path loss models and time dispersion in an outdoor line-of-sight environment for $5 \mathrm{G}$ wireless communications by Ahmed M.Al-Samman, Tharek A.Rahman, Marwan H.Azmi, M.N.Hindia, 2016. 
Figures

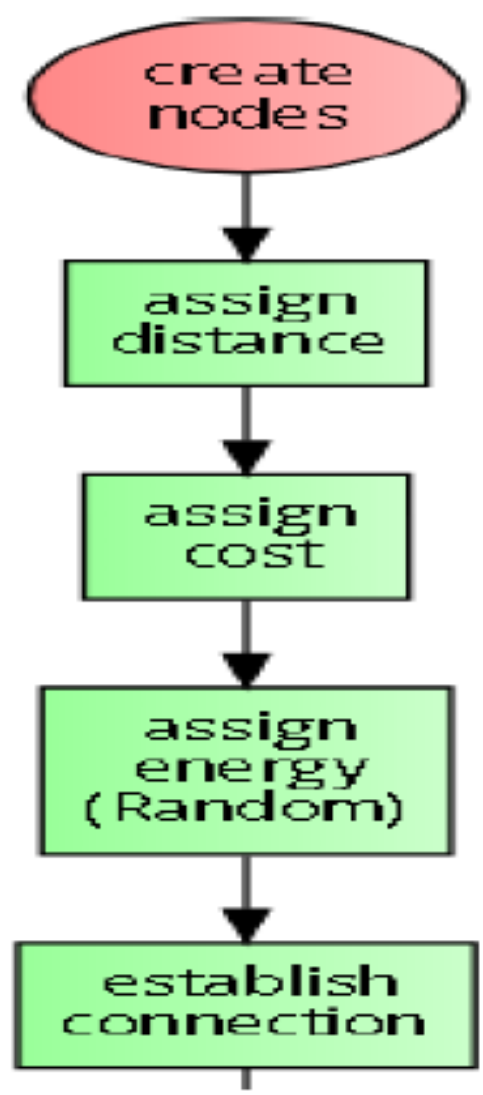

Figure 1

Module 1: Design of wireless sensor networks

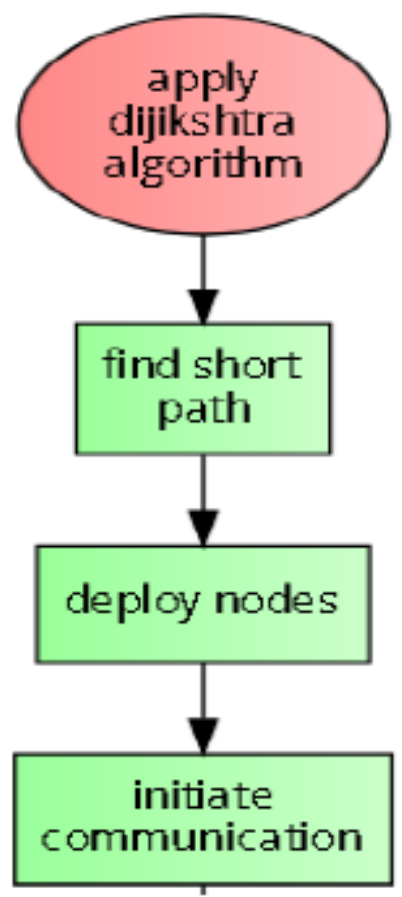

Figure 2 
Module 2: Communication Establishment

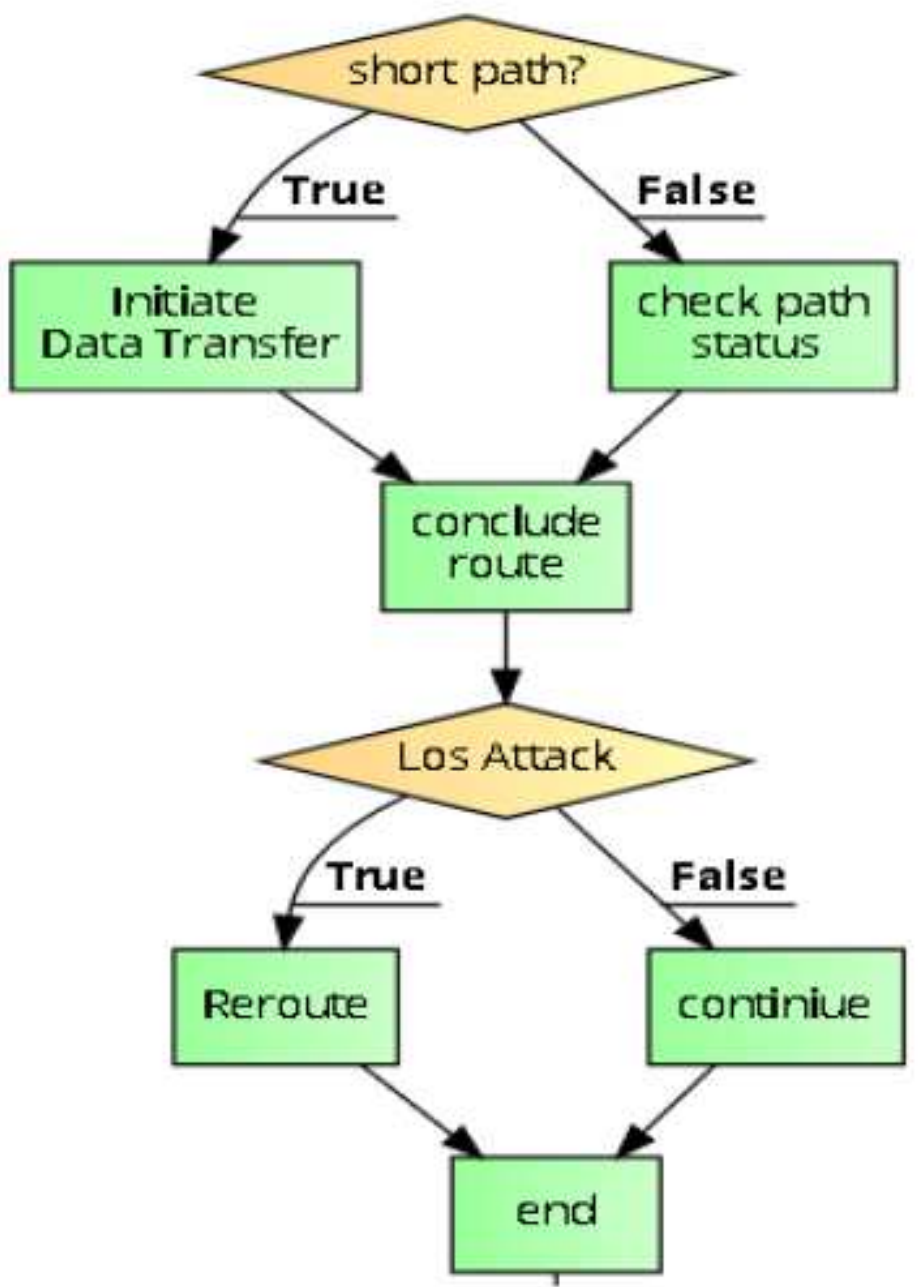

Figure 3

Module 3: RNN for Abnormality Analysis 

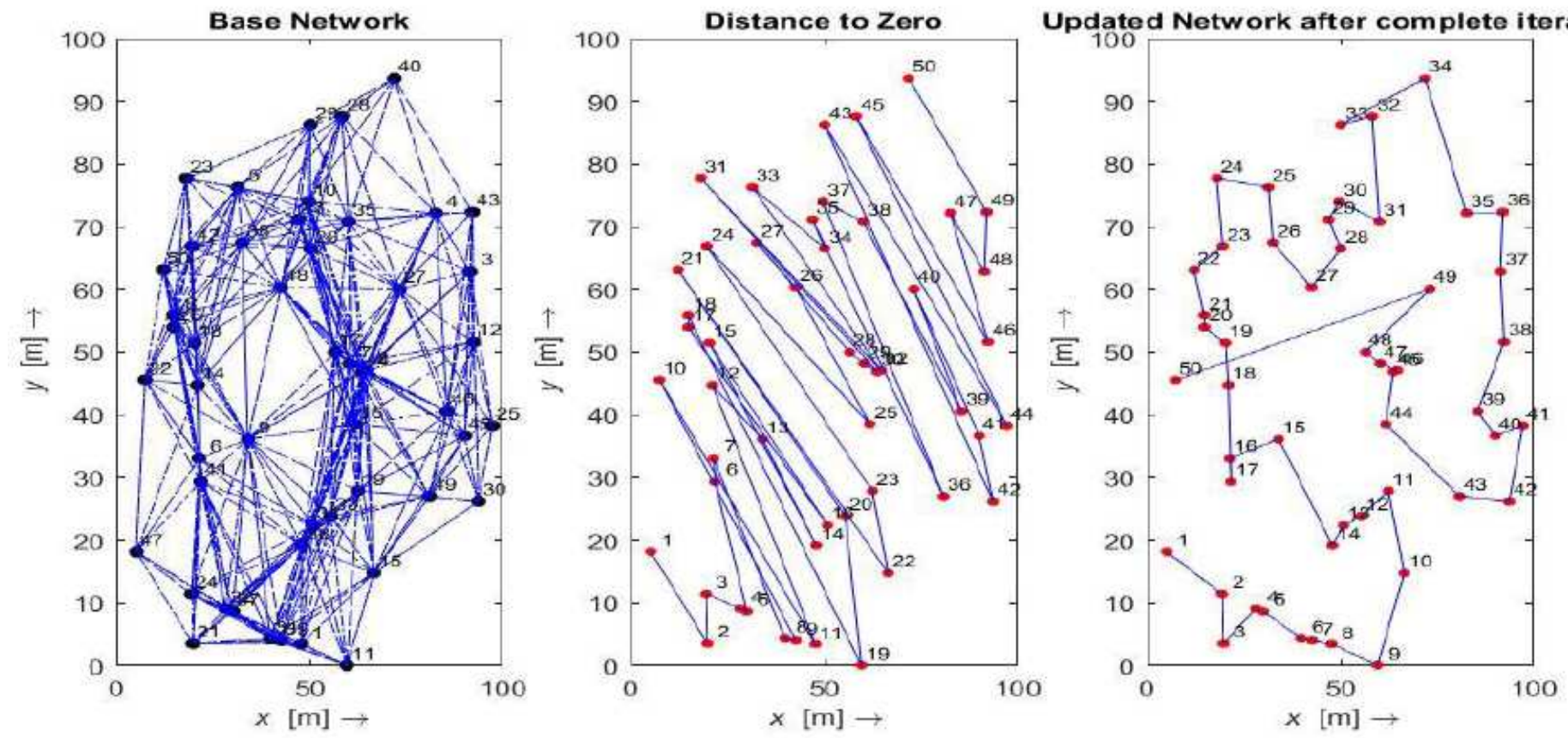

\section{Figure 4}

Process of Node Connectivity

\section{Network}

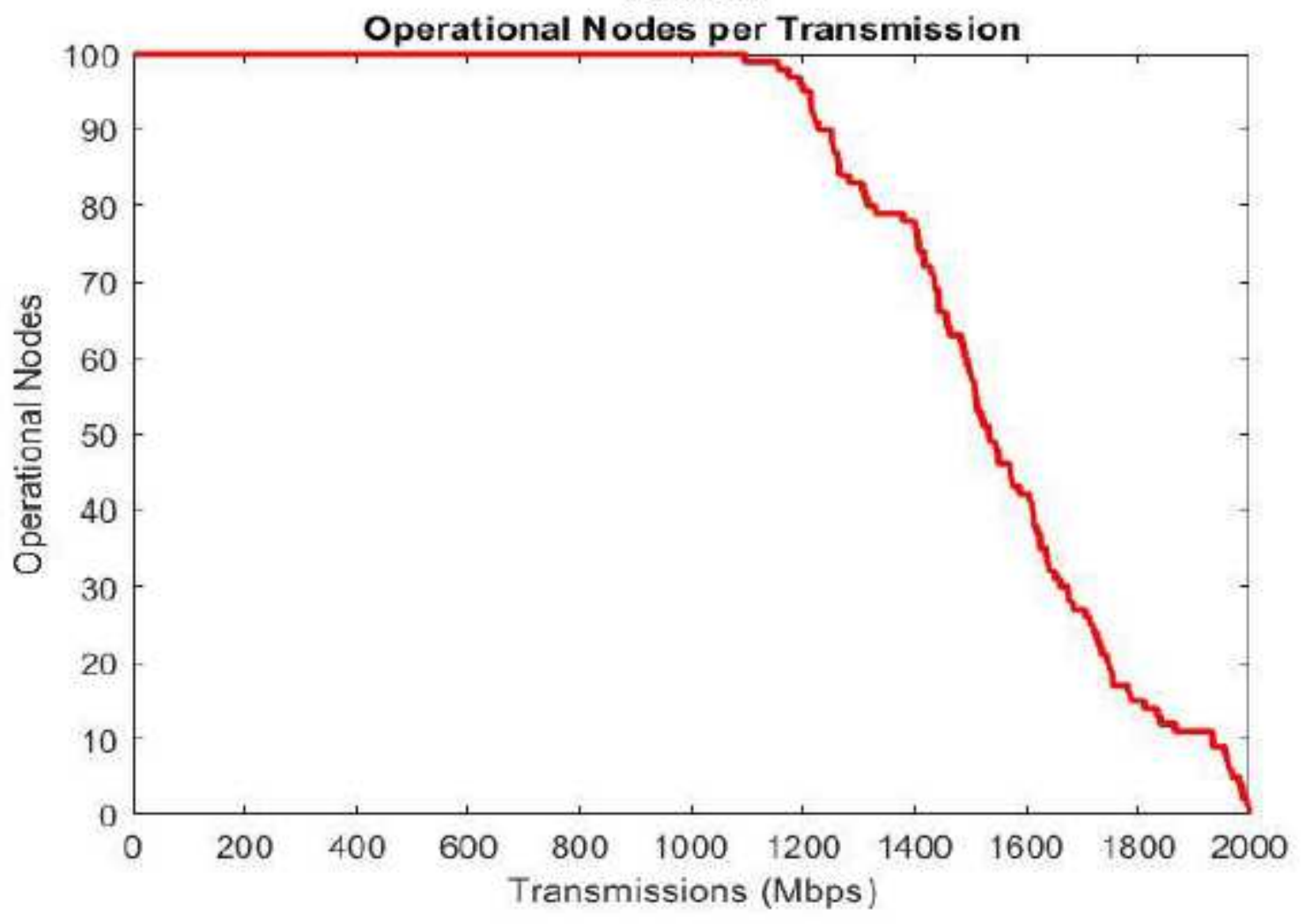

Figure 5 


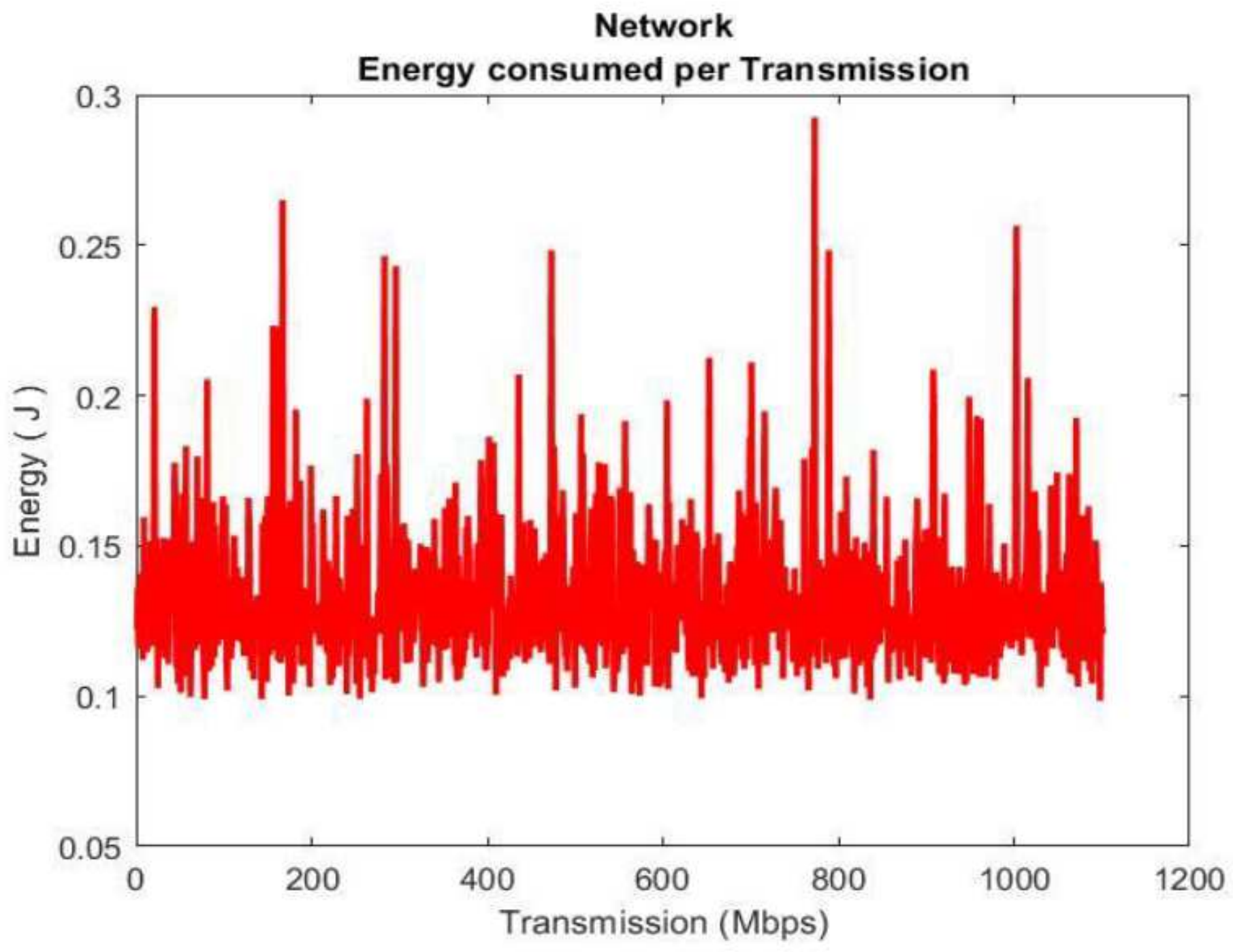

Figure 6

Energy Consumed per Transmission 


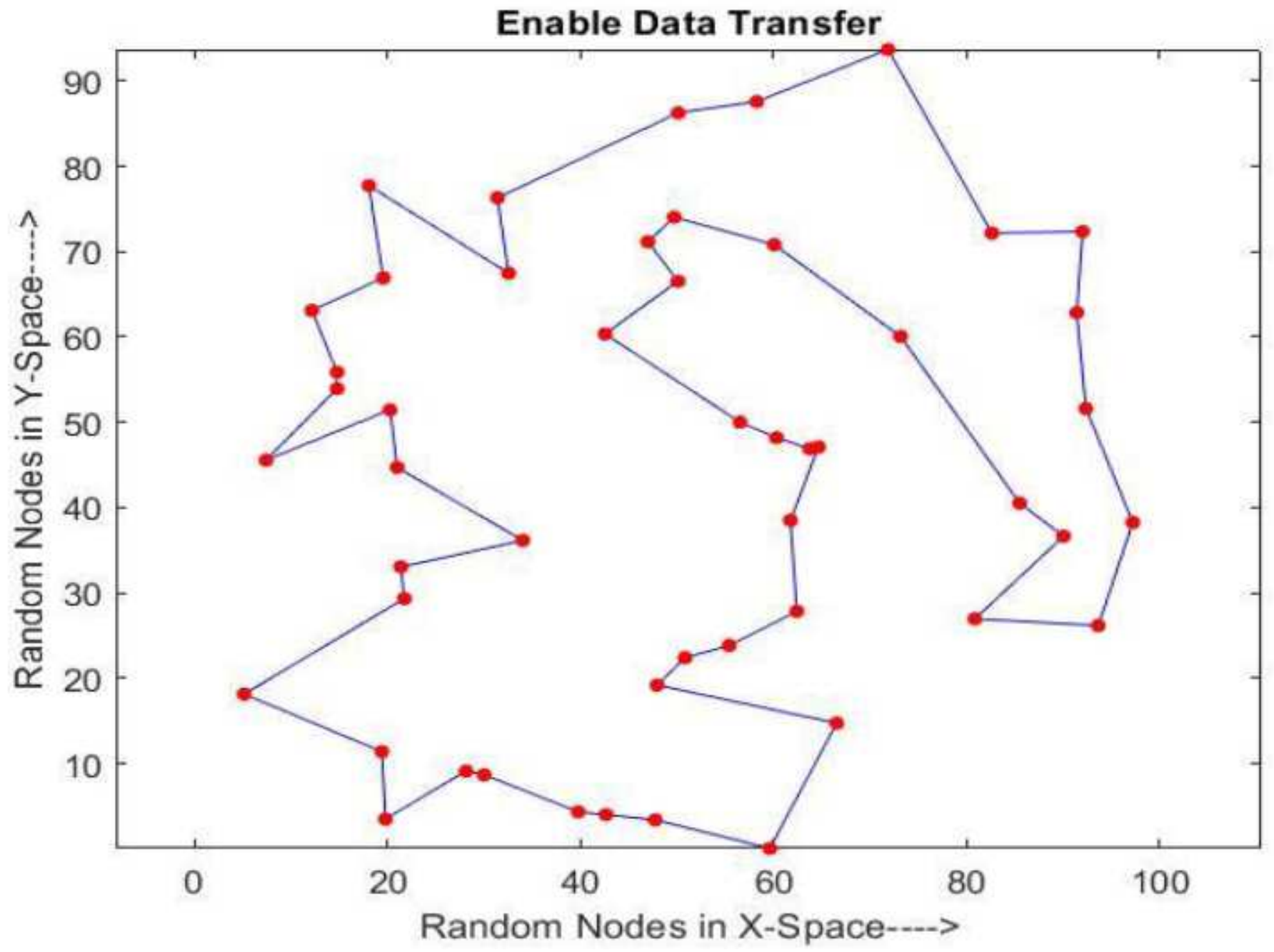

Figure 7

Enable Data Transfer 


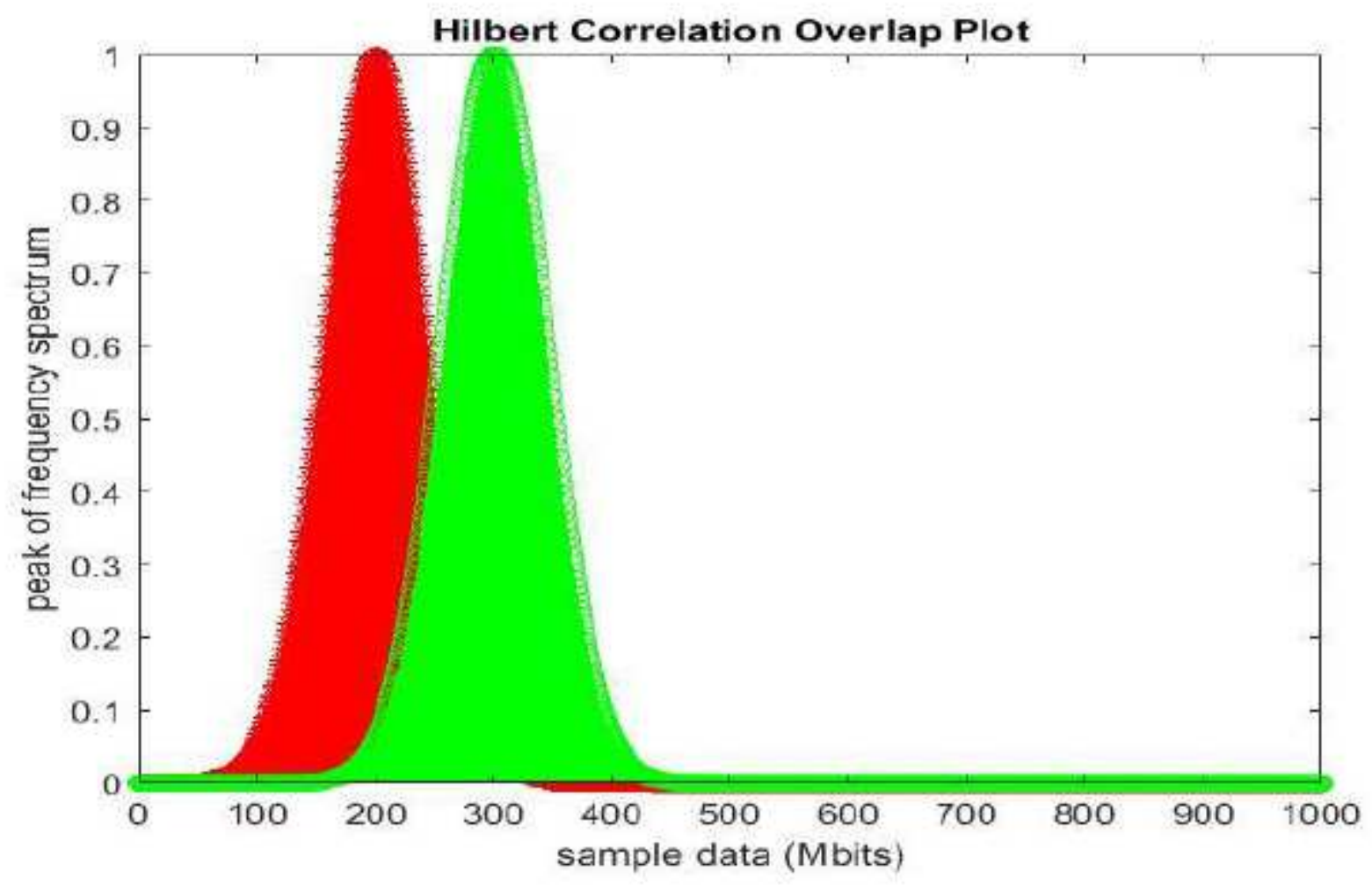

Figure 8

Hilbert Correlation Overlap Plot

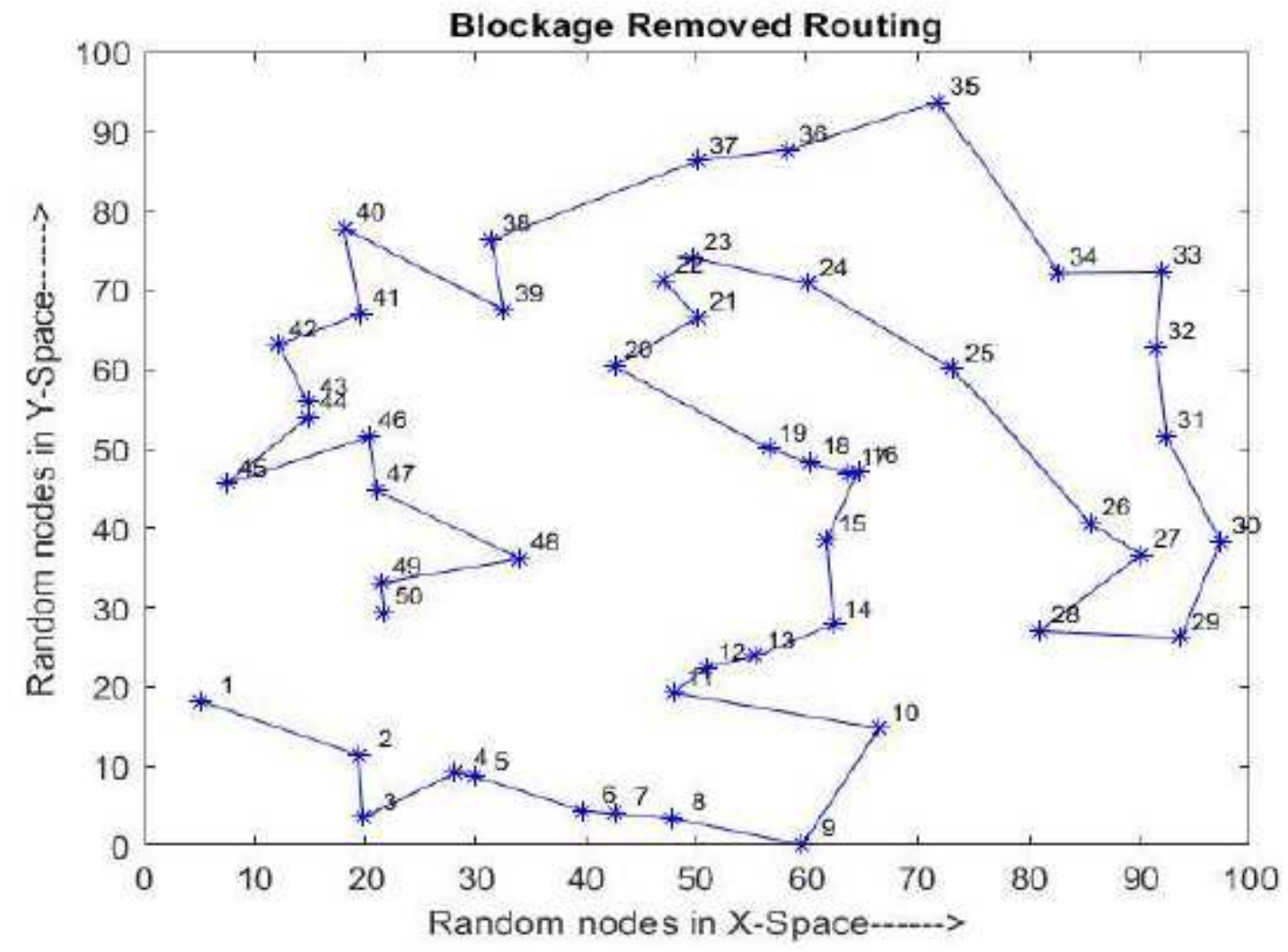


Figure 9

Blockage Removed Routing 